\title{
Berberine-induced anticancer activities in FaDu head and neck squamous cell carcinoma cells
}

\author{
YO-SEOB SEO ${ }^{1}$, MIN-JI YIM ${ }^{2}$, BOK-HEE KIM ${ }^{2}$, KYUNG-ROK KANG ${ }^{2}$, SOOK-YOUNG LEE ${ }^{2}$, \\ JI-SU OH ${ }^{3}$, JAE-SEEK YOU ${ }^{3}$, SU-GWAN KIM ${ }^{2,3}$, SANG-JOUN YU ${ }^{4}$, GYEONG-JE LEE ${ }^{5}$, \\ DO KYUNG KIM ${ }^{6}$, CHUN SUNG KIM ${ }^{6}$, JIN-SOO KIM ${ }^{1}$ and JAE-SUNG KIM ${ }^{7}$
}

\author{
${ }^{1}$ Department of Oral and Maxillofacial Radiology, ${ }^{2}$ Regional Innovation Center for Dental Science and Engineering, \\ ${ }^{3}$ Department of Oral and Maxillofacial Surgery, ${ }^{4}$ Department of Periodontology, ${ }^{5}$ Department of Prosthodontics, \\ ${ }^{6}$ Oral Biology Research Institute, ${ }^{7}$ Pre-Dentistry, School of Dentistry, Chosun University, \\ Gwang-Ju 501-759, Republic of Korea
}

Received June 30, 2015; Accepted August 10, 2015

DOI: $10.3892 /$ or.2015.4312

\begin{abstract}
In the present study, we investigated berberine-induced apoptosis and the signaling pathways underlying its activity in FaDu head and neck squamous cell carcinoma cells. Berberine did not affect the viability of primary human normal oral keratinocytes. In contrast, the cytotoxicity of berberine was significantly increased in FaDu cells stimulated with berberine for $24 \mathrm{~h}$. Furthermore, berberine increased nuclear condensation and apoptosis rates in $\mathrm{FaDu}$ cells than those in untreated control cells. Berberine also induced the upregulation of apoptotic ligands, such as FasL and TNF-related apoptosis-inducing ligand, and triggered the activation of caspase- $8,-7$ and -3 , and poly(ADP ribose) polymerase, characteristic of death receptor-dependent extrinsic apoptosis. Moreover, berberine activated the mitochondria-dependent apoptotic signaling pathway by upregulating pro-apoptotic factors, such as Bax, Bad, Apaf-1, and the active form of caspase- 9 , and downregulating anti-apoptotic factors, such as Bcl-2 and Bcl-xL. In addition, berberine increased the expression of the tumor suppressor p53 in FaDu cells. The pan-caspase inhibitor Z-VAD-fmk suppressed the activation of caspase- 3 and prevented cytotoxicity in $\mathrm{FaDu}$ cells treated with berberine. Interestingly, berberine suppressed cell migration through downregulation of vascular endothelial growth factor (VEGF), matrix metalloproteinase (MMP)-2, and MMP-9. Moreover, the phosphorylation of extracellular signal-regulated kinase (ERK1/2) and p38, components of the mitogen-activated protein kinase pathway that are associated
\end{abstract}

Correspondence to: Professor Jae-Sung Kim, Pre-Dentistry, School of Dentistry, Chosun University, 309 Philmun-daero, Dong-gu, Gwang-Ju 501-759, Republic of Korea

E-mail: js_kim@chosun.ac.kr

Key words: berberine, head and neck squamous cell carcinoma, FaDu cells, apoptosis, chemoprevention with the expression of MMP and VEGF, was suppressed in FaDu cells treated with berberine for $24 \mathrm{~h}$. Therefore, these data suggested that berberine exerted anticancer effects in $\mathrm{FaDu}$ cells through induction of apoptosis and suppression of migration. Berberine may have potential applications as a chemotherapeutic agent for the management of head and neck squamous carcinoma.

\section{Introduction}

Head and neck squamous carcinoma (HNSCC) is a common cancer associated with high mortality, accounting for $\sim 3-5 \%$ of all human malignancies. Owing to the increasing consumption of alcohol $(1,2)$, tobacco $(2,3)$, and betel nut chewing (4) and increased rates of opportunistic infection with human papilloma virus (5), $\sim 640,000$ cases of HNSCC are reported worldwide each year $(6,7)$. Despite improvements in clinical interventions, including surgery, radiotherapy, chemotherapy, and chemoradiotherapy, the 5-year survival rate in patients with HNSCC has not improved within the last several decades (8). Additionally, survival and morbidity rates in patients with HNSCC have not improved significantly in the last 30 years. While chemotherapy is thought to be one of the primary clinical management strategies for HNSCC, the efficacy and safety of clinical chemotherapeutic agents are not sufficient. Therefore, recent strategies for developing chemotherapeutic agents have focused on achieving cancer cell-specific apoptosis without affecting normal cells (9).

Apoptosis, a process involving programmed cell death, is an essential physiological process required for normal tissue development and homeostasis (10). Recently, induction of apoptosis through induction of DNA damage in cancer cells has been considered as an effective anticancer strategy (11). Furthermore, based on our understanding of apoptotic signaling pathways associated with cancer cell-specific death, molecules targeting apoptotic pathways have been tested in preclinical and clinical studies (11). However, currently available chemotherapeutic agents that target molecules associated with apoptosis have several clinical limitations, such as low efficacy in cancer cells, high cytotoxicity in normal cells, and 
induction of chemoresistance (12). Therefore, recent studies associated with the development of chemotherapeutic agents for cancer therapy have explored the efficacy and safety of natural compounds from herbal plants.

Berberine $\left(\mathrm{C}_{20} \mathrm{H}_{18} \mathrm{NO}_{4}\right)$ is a type of isoquinoline alkaloid purified from Rhizoma coptidis and Cortex phellodendri. Recent pharmacological studies of berberine have demonstrated that this molecule alleviates diabetic nephropathy (13), rheumatoid arthritis (14), and inflammation (15). Moreover, berberine has antioxidant effects $(16,17)$, prevents obesity (18), and has anticancer activities in breast (19), prostate (20), cervical (21), gastric (22), oral $(23,24)$, and ovarian cancers $(25)$. Although these anticancer effects of berberine and associated cell signaling pathways have been reported in various cancers, the effects of berberine on apoptosis have not been reported in HNSCC.

Therefore, the aim of this study is to determine whether berberine could function as a chemotherapeutic agent in HNSCC. Furthermore, we evaluated the effects of berberine on apoptotic signaling pathway in HNSCC.

\section{Materials and methods}

Cell culture. FaDu cells, a human pharyngeal squamous carcinoma cell line, were obtained from the American Type Culture Collection (ATCC) and cultured according to the instructions provided by the ATCC. Briefly, FaDu cells were maintained in minimum essential medium (Life Technologies, Grand Island, NY, USA) containing $10 \%$ fetal bovine serum (FBS). Cells were grown in a humidified incubator at $37^{\circ} \mathrm{C}$ in an atmosphere containing $5 \% \mathrm{CO}_{2}$.

Cell cytotoxicity. The cells were cultured at a density of $1 \times 10^{5}$ cells $/ \mathrm{ml}$ in $96-$ well culture plates for $24 \mathrm{~h}$ in a humidified incubator at $37^{\circ} \mathrm{C}$ in an atmosphere containing $5 \% \mathrm{CO}_{2}$. Cultured FaDu cells were treated with 12.5 or $25 \mu \mathrm{M}$ berberine for $24 \mathrm{~h}$. Thereafter, $20 \mu \mathrm{l}$ of $5 \mathrm{mg} / \mathrm{ml} \mathrm{3-(4,5-dimethylthi-}$ azol-2-yl)-2,5-diphenyltetrazolium bromide (MTT) was added to the cultured FaDu cells in the presence of berberine. After $4 \mathrm{~h}$, the supernatant was removed, and the formazan crystals formed from reaction with MTT were dissolved in dimethyl sulfoxide $(200 \mu \mathrm{l} /$ well). Finally, the optical density was measured at $570 \mathrm{~nm}$ using a spectrometer. Experiments were performed at least three times.

Cell survival assay. Cell survival was measured as previously described (26), using Green calcein-AM and ethidium homodimer-1 (Life Technologies) to stain viable and dead cells, respectively. To evaluate cell survival, FaDu cells were plated on chamber slides and treated with 12.5 or $25 \mu \mathrm{M}$ berberine for $24 \mathrm{~h}$. Thereafter, cell survival assays were performed according to the manufacturer's protocol. Cells were then examined and imaged using a fluorescence microscope (Eclipse TE200; Nikon Instruments, Melville, NY, USA).

4',6-Diamidino-2-phenylindole (DAPI) staining. To observe nuclear condensation associated with apoptosis, FaDu cells treated with 12.5 or $25 \mu \mathrm{M}$ berberine for $24 \mathrm{~h}$ were fixed with $4 \%$ paraformaldehyde prior to washing with phosphate-buffered saline (PBS). The cells were then stained with
$1 \mathrm{mg} / \mathrm{ml}$ DAPI (Life Technologies) for $20 \mathrm{~min}$ in the dark. Nuclear condensation was observed by fluorescence microscopy (Eclipse TE200; Nikon Instruments).

Quantification of apoptosis using flow cytometric analysis. Flow cytometric analysis was performed in cells co-stained with Annexin V and propidium iodide (PI; Cell Signaling Technology, Danvers, MA, USA) to detect apoptosis. First, $\mathrm{FaDu}$ cells were plated at $5 \times 10^{5}$ cells $/ \mathrm{ml}$ in 6 -well plates and incubated for $24 \mathrm{~h}$ in a humidified incubator at $37^{\circ} \mathrm{C}$ with $5 \% \mathrm{CO}_{2}$. Thereafter, the cells were treated with $25 \mu \mathrm{M}$ berberine for $24 \mathrm{~h}$. Both floating and attached cells were then collected, washed twice with ice-cold PBS, and resuspended in $500 \mu \mathrm{l}$ of $1 \mathrm{X}$ binding buffer (BD Biosciences, San Diego, CA, USA). Annexin V and PI were added to the cells, and cells were incubated for $15 \mathrm{~min}$ at $37^{\circ} \mathrm{C}$ in the dark. The population of Annexin V-positive cells and the cell cycle distribution were analyzed using a BD CellQuest version 3.3 instrument (Becton-Dickinson, San Jose, CA, USA) and WinMDI version 2.9 software (The Scripps Research Institute, San Diego, CA, USA).

Western blot analysis. FaDu cells were plated at a density of $5 \times 10^{6}$ cells $/ \mathrm{ml}$ in culture dishes and incubated for $24 \mathrm{~h}$ in a humidified incubator at $37^{\circ} \mathrm{C}$ with $5 \% \mathrm{CO}_{2}$. Cultured $\mathrm{FaDu}$ cells were treated with 12.5 or $25 \mu \mathrm{M}$ berberine for $24 \mathrm{~h}$. Thereafter, cells were harvested, lysed using cell lysis buffer (Cell Signaling Technology) containing protease and phosphatase inhibitor cocktails, and incubated for $1 \mathrm{~h}$ at $4^{\circ} \mathrm{C}$. Lysates were centrifuged at $14,000 \mathrm{x}$ for $10 \mathrm{~min}$ at $4^{\circ} \mathrm{C}$. The supernatant was used as the cytosolic fraction. Total protein concentrations of the cell lysates were determined by bicinchoninic acid protein assays (Thermo Scientific, Rockford, IL, USA). Next, 5X loading buffer was added to equal amounts of protein, and the mixtures were boiled at $90^{\circ} \mathrm{C}$ for $10 \mathrm{~min}$. Total proteins were separated using sodium dodecyl sulfate-polyacrylamide gel electrophoresis (SDS-PAGE) and transferred to nitrocellulose membranes. After blocking for $2 \mathrm{~h}$ with $5 \%$ bovine serum albumin in Tris-buffered saline containing Tween-20 at room temperature, membranes were incubated with primary antibodies at $4^{\circ} \mathrm{C}$ overnight and then incubated with horseradish peroxidase-conjugated secondary antibodies. The following antibodies (purchased from Cell Signaling Technology) were used to verify the apoptotic signaling pathways: anti-Fas ligand (FasL; $48 \mathrm{kDa}$ ), antitumor necrosis factor-related apoptosis-inducing ligand (TRAIL; $28 \mathrm{kDa}$ ), anti-cleaved caspase-3 (17 and $19 \mathrm{kDa}$ ), anticleaved caspase-7 (18 kDa), anti-cleaved caspase-8 (18 kDa), anti-cleaved caspase-9 (37 kDa), anti-poly(ADP ribose) polymerase (PARP; full-length form, $116 \mathrm{kDa}$, cleaved form, $85 \mathrm{kDa})$, anti-p53 (53 kDa), anti-B-cell lymphoma 2 (Bcl-2; $26 \mathrm{kDa}$ ), anti-Bcl extra-large (Bcl-xL; $26 \mathrm{kDa})$, anti-Bcl2-associated X protein (Bax; $21 \mathrm{kDa}$ ), anti-Bcl-2-associated death promoter (Bad; $23 \mathrm{kDa})$, and anti-apoptotic proteaseactivating factor 1 (Apaf-1; $13 \mathrm{kDa}$ ). Furthermore, antibodies associated with mitogen-activated protein kinase (MAPK) signaling (Cell Signaling Technology) were used as follows: anti-phospho-extracellular signal-regulated kinase (ERK)1/2 (42 and $44 \mathrm{kDa}$ ), anti-ERK1/2 (42 and $44 \mathrm{kDa}$ ), anti-phosphop38 (38 kDa), anti-p38 (38 kDa), anti-phospho-c-Jun $\mathrm{N}$ 
terminal kinase (JNK; 46 and $54 \mathrm{kDa}$ ), anti-JNK (46 and $54 \mathrm{kDa})$, anti-phospho-Akt (60 kDa), anti-Akt (60 kDa), and anti- $\beta$-actin (42 kDa). Antibodies against matrix metalloproteinase (MMP)-2 (63 kDa), MMP-9 (92 kDa), and vascular endothelial growth factor (VEGF; $21 \mathrm{kDa}$ ), from Santa Cruz Biotechnology (Dallas, TX, USA), were used to evaluate the suppression of $\mathrm{FaDu}$ cell migration in the presence of berberine. The immunoreactive bands were visualized using the ECL system (Amersham Biosciences, Piscataway, NJ, USA) and exposure to radiographic film.

Caspase-3/-7 activity assay. The apoptotic activities of executioner caspases-3 and -7 were determined using the cell-permeable fluorogenic substrate PhiPhiLux- $\mathrm{G}_{1} \mathrm{D}_{2}$ (OncoImmunin Inc., Gaithersburg, MD, USA), according to the manufacturer's instructions.

Gelatin zymography. Equal volumes of cell culture supernatants were mixed with non-reducing sample buffer [4\% SDS, $0.15 \mathrm{M}$ Tris ( $\mathrm{pH}$ 6.8), and $20 \%$ glycerol containing $0.05 \%$ bromophenol blue] and resolved on $10 \%$ polyacrylamide gels containing copolymerized $0.2 \%(1 \mathrm{mg} / \mathrm{ml})$ swine skin gelatin (Sigma-Aldrich, St. Louis, MO, USA). After electrophoresis of the conditioned medium supernatant samples, gels were washed twice for 15 min each with $2.5 \%$ Triton X-100. Digestion was carried out by incubating the gel in gelatinase buffer [50 mM Tris- $\mathrm{HCl}(\mathrm{pH} 7.6), 10 \mathrm{mM} \mathrm{CaCl} 2,50 \mathrm{mM}$ $\mathrm{NaCl}$, and $0.05 \% \mathrm{Brij}-35]$ at $37^{\circ} \mathrm{C}$ for $24 \mathrm{~h}$. The gel was stained with $0.1 \%$ Coomassie Brilliant Blue R-250 (GE Healthcare, Piscataway, NJ, USA), and the locations of gelatinolytic activity were revealed as clear bands on a background of uniform light blue staining.

Migration assay. To perform migration assays, FaDu cells were cultured on culture inserts $\left(2 \times 0.22 \mathrm{~cm}^{2}\right.$; Ibidi, Regensburg, Germany) at $1 \times 10^{4}$ cells/well. Wounds were introduced by removing the culture inserts after $24 \mathrm{~h}$ of incubation. The width of the wound was measured using images obtained with an inverted microscope.

Quantitative polymerase chain reaction ( $q P C R)$. FaDu cells were plated at a density of $5 \times 10^{6}$ cells $/ \mathrm{ml}$ on culture dishes and incubated for $24 \mathrm{~h}$ at $37^{\circ} \mathrm{C}$ in an atmosphere containing $5 \%$ $\mathrm{CO}_{2}$. Cultured FaDu cells were treated with 12.5 or $25 \mu \mathrm{M}$ berberine for $24 \mathrm{~h}$. Thereafter, total RNA was isolated using TRIzol reagent (Life Technologies) according to the manufacturer's instructions. Total RNA $(1 \mu \mathrm{g})$ was reverse transcribed into first-strand cDNA using the ThermoScript RT-PCR system (Life Technologies). For qPCR, cDNA was amplified using a SureCycler 8800 (Agilent Technologies, Santa Clara, CA, USA) and 2X TOPsimple DyeMIX-nTaq (Enzynomics, Seoul, Korea), according to the instructions of the manufacturers. Gene expression was determined by agarose gel electrophoresis. Glyceraldehyde 3-phosphate dehydrogenase (GAPDH) was used as the internal control for normalization. The following primers were used for qPCR: $M M P-2$ forward, 5'-GGACAAGAACCAGATCACATACA-3' and reverse, 5'-CATACTTCACACGGACCACTT-3' (human MMP-2; PCR product size: 320 bp; NCBI accession no. NM_001302510.1); MMP-9 forward, 5'-GAGAACCAATCTCACCGACAG-3' and reverse, 5'-GACACCAAACTGGATGACGA-3' (human MMP-9; PCR product size, 412 bp; NCBI accession no. NM_004994.2); and GAPDH forward,5'-CTTTGGTATCGTG GAAGGACTC-3' and reverse, 5'-CCTGCTTCACCACCTT CTT-3' (human GAPDH; PCR product size, 293 bp; NCBI accession no. NM_001289746.1). After PCR, amplified PCR products were visualized by DNA agarose gel electrophoresis. The differences in expression levels are presented as histograms after densitometry analysis using a VersaDoc imaging system (Bio-Rad, Hercules, CA, USA).

Caspase-dependent cell survival assay. The cells were plated at a density of $1 \times 10^{5}$ cells $/ \mathrm{ml}$ in 96 -well plates and allowed to attach overnight. After incubation, cultured cells were treated with 12.5 or $25 \mu \mathrm{M}$ berberine in the presence or absence of $50 \mu \mathrm{M}$ Z-VAD-fmk [N-benzyloxycarbonyl-Val-Ala-Asp (O-Me) fluoromethyl ketone], a caspase-3 inhibitor (Sigma-Aldrich) and were incubated for $24 \mathrm{~h}$ at $37^{\circ} \mathrm{C}$. The cytotoxicity of berberine was then measured by MTT assay.

Statistical analysis. Data are reported as the mean \pm SD of three individual experiments performed in triplicate. Statistical analysis was carried out using Student's t-tests, and differences with p-values of $<0.05$ were considered statistically significant.

\section{Results}

Berberine exhibited cytotoxicity in FaDu cells. To determine whether berberine was cytotoxic to HNSCC cells, the viability of FaDu cells was measured by MTT assay at $24 \mathrm{~h}$ after treatment with 12.5 or $25 \mu \mathrm{M}$ berberine. As shown in Fig. 1A, the viability of FaDu cells was decreased by $\sim 37 \%(\mathrm{p}<0.01)$ and $45 \%(\mathrm{p}<0.01)$ by 12.5 and $25 \mu \mathrm{M}$ berberine, respectively, compared with that in untreated control cells. Furthermore, to confirm the viability of FaDu cells in presence of 12.5 or $25 \mu \mathrm{M}$ berberine, we performed assays for viable and dead using cell-permeable Green calcein-AM to stain live cells (green fluorescence) and ethidium homodimer-1 to stain dead cells (red fluorescence). As shown in Fig. 1B, the density of FaDu cells treated with 12.5 or $25 \mu \mathrm{M}$ berberine for $24 \mathrm{~h}$ was reduced compare with that in untreated control cells. Moreover, the number of dead cells stained with ethidium homodimer-1 was increased in FaDu cells treated with berberine. Therefore, these data suggested that berberine was cytotoxic to $\mathrm{FaDu}$ cells in a concentration-dependent manner.

Berberine-induced FaDu cell death was associated with apoptosis. To determine whether the apoptotic pathway was involved in the observed berberine-induced $\mathrm{FaDu}$ cell death, FaDu cells were treated with 12.5 or $25 \mu \mathrm{M}$ berberine for $24 \mathrm{~h}$, and nuclear staining with DAPI was performed to visualize nucleus condensation, a typical feature of cells undergoing apoptosis. As shown in Fig. 1C, berberine treatment caused a decrease in the density of FaDu cells compared with that in untreated control cells, consistent with the results of the viable and dead cell assay. Furthermore, the number of $\mathrm{FaDu}$ cells with condensed nuclei was increased by berberine treatment in a concentration-dependent manner. Next, to further verify the effects of berberine on apoptosis in FaDu cells, we performed FACS analysis using PI and Annexin V staining. 
A

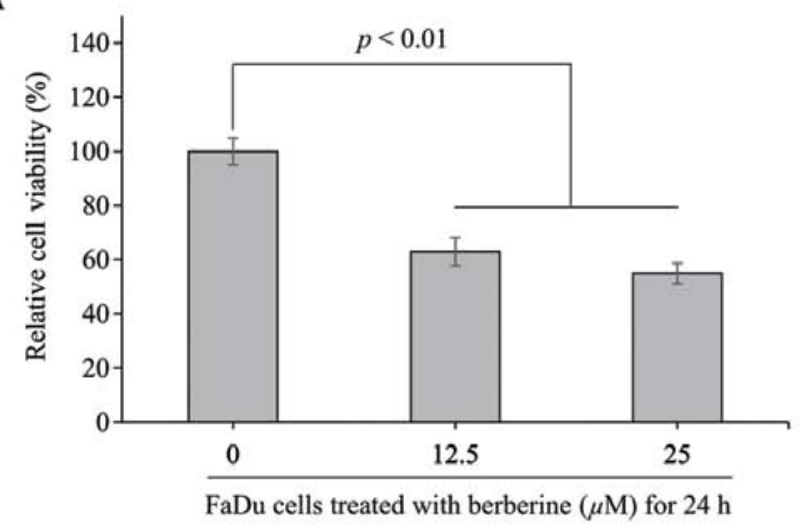

C

FaDu cells treated with berberine $(\mu \mathrm{M})$ for $24 \mathrm{~h}$

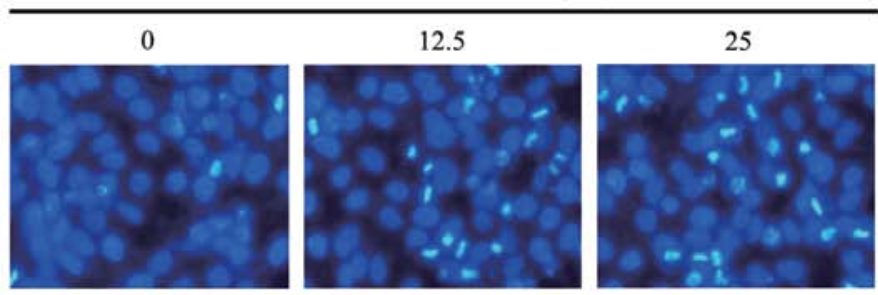

B FaDu cells treated with berberine $(\mu \mathrm{M})$ for $24 \mathrm{~h}$

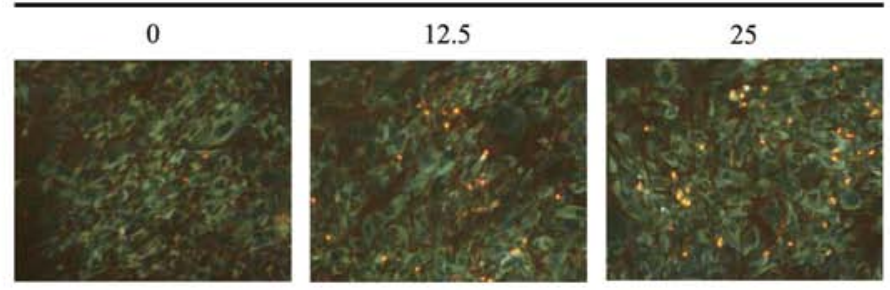

D
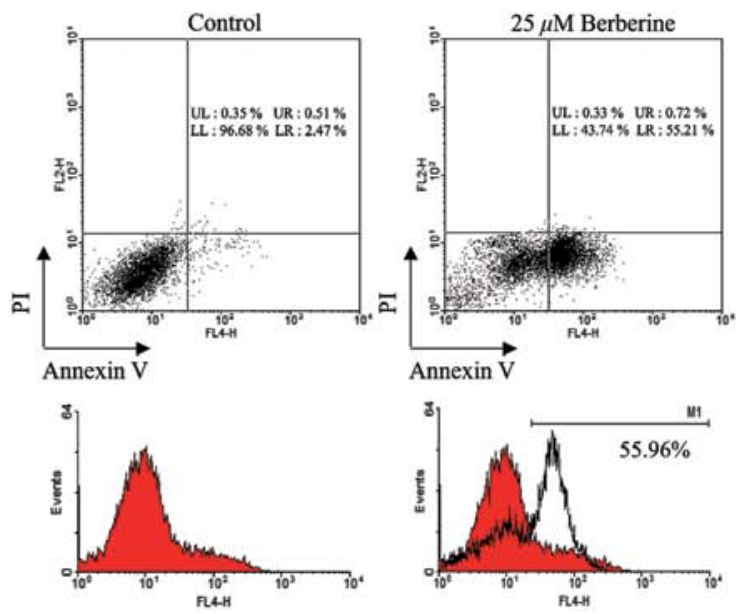

Figure 1. Berberine increased FaDu cell death through apoptosis. (A) Berberine increased FaDu cell death in a concentration-dependent manner. (B) The population of dead cells was gradually increased in FaDu cells treated with berberine in a concentration-dependent manner. (C) Berberine induced chromatin condensation in FaDu cells. (D) The apoptotic population was increased in FaDu cells treated with berberine.

As shown in Fig. 1D, the population of apoptotic cells was significantly increased by $\sim 54.29 \%$ in FaDu cells treated with $25 \mu \mathrm{M}$ berberine for $24 \mathrm{~h}$ compared with that in untreated control cells. Taken together, these data further supported that berberine induced cytotoxic effects through an apoptotic pathway in FaDu cells.

Both death receptor-dependent extrinsic and mitochondria-dependent intrinsic apoptotic signaling pathways were involved in berberine-induced apoptosis in FaDu cells. Next, to elucidate the apoptotic signaling pathways involved in berberine-induced apoptosis in FaDu cells, we measured the expression of death receptor ligands, such as FasL and TRAIL, by western blotting in FaDu cells treated with 12.5 or $25 \mu \mathrm{M}$ berberine for $24 \mathrm{~h}$. As shown in Fig. 2A, berberine significantly increased the expression of FasL and TRAIL in FaDu cells. Subsequently, both cleaved caspase- 8 and cleaved caspase-7 were significantly increased in FaDu cells treated with berberine in a concentration-dependent manner. These data clearly suggested that the death receptor-dependent extrinsic apoptotic signaling pathway was involved in berberine-induced apoptosis in FaDu cells. Furthermore, the expression levels of anti-apoptotic factors Bcl-2 and Bcl-xL were significantly decreased in $\mathrm{FaDu}$ cells treated with berberine in a concentration-dependent manner, as shown in Fig. 2B. Conversely, the expression levels of pro-apoptotic factors Bax, Bad, Apaf-1, and cleaved caspase-9 were significantly increased by berberine in FaDu cells. These data indicated that the mitochondria-dependent intrinsic apoptotic signaling pathway was triggered by cleaved caspase- 8 and was involved in berberine-induced apoptosis in FaDu cells. However, caspase-3 is a target molecule of both death receptordependent extrinsic and mitochondria-dependent intrinsic apoptosis. Therefore, we determined the expression of cleaved caspase-3 and its downstream target molecule PARP. As shown in Fig. 2C, cleaved caspase-3 was significantly upregulated in FaDu cells treated with berberine. Subsequently, levels of both the pro-form and cleaved form of PARP, which functions downstream of caspase-3, were significantly increased by berberine treatment in FaDu cells. To further confirm the activation of caspase- 3 in FaDu cells treated with berberine, we performed PhiphiLux-caspase-3/-7 assays. As shown in Fig. 2D, the population of FaDu cells stained with green, representing cleaved caspase-3/-7, was increased following berberine treatment in a concentration-dependent manner. Taken together, these data indicated that berberine-induced apoptosis in FaDu cells was mediated by the activation of caspase- 3 through both the death receptor-dependent extrinsic pathway and the mitochondria-dependent intrinsic apoptotic signaling pathway.

Berberine-induced apoptosis in FaDu cells was regulated by caspase activation. Apoptotic signaling pathways are mediated by the activation of the caspase cascade, a hallmark of apoptosis. Therefore, to determine whether berberine-induced apoptosis in $\mathrm{FaDu}$ cells was dependent on the activation of caspases, FaDu cells were pretreated for 30 min with $50 \mu \mathrm{M}$ Z-VAD-fmk, a pan-caspase inhibitor, prior to treatment with $25 \mu \mathrm{M}$ berberine. MTT assays were then performed to measure cell viability, and western blotting was used to observe changes 
$\mathbf{A}$

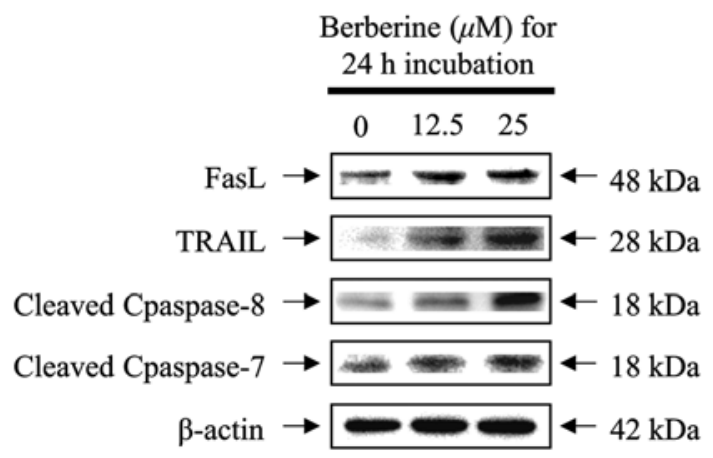

C

Berberine $(\mu \mathrm{M})$ for $24 \mathrm{~h}$ incubation

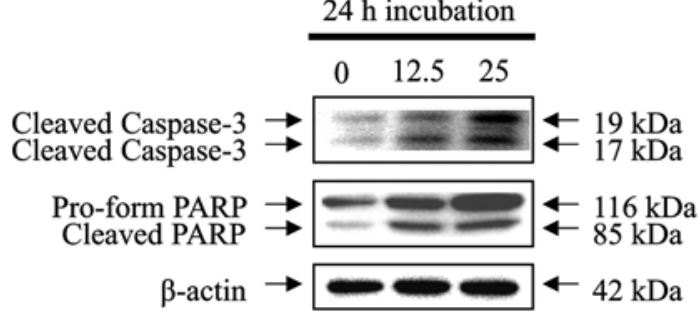

B

Berberine $(\mu \mathrm{M})$ for

$24 \mathrm{~h}$ incubation

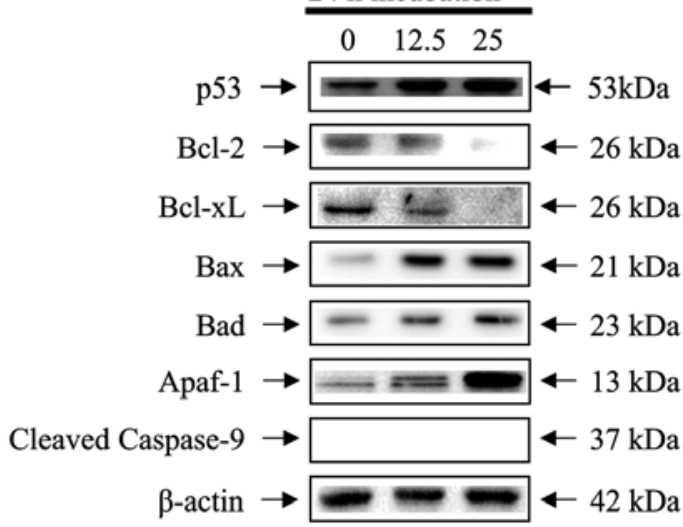

D

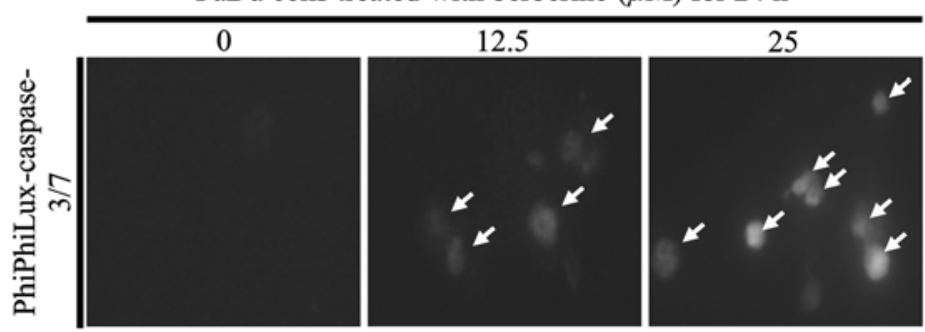

Figure 2. Berberine-induced apoptosis in FaDu cells was mediated by both death receptor-dependent extrinsic and mitochondria-dependent intrinsic apoptotic signaling pathways. (A) Berberine triggered the death receptor-dependent extrinsic apoptotic signaling pathway through the upregulation of death receptor ligands in FaDu cells. (B) Berberine-induced apoptosis in FaDu cells was involved in the mitochondria-dependent intrinsic apoptotic signaling pathway. (C) Activation of caspase-3 and PARP via berberine-induced death receptor-dependent extrinsic and mitochondria-dependent intrinsic apoptotic signaling pathways. (D) PhiPhiLux-caspase-3/-7 substrate was cleaved in FaDu cells treated with berberine.

A

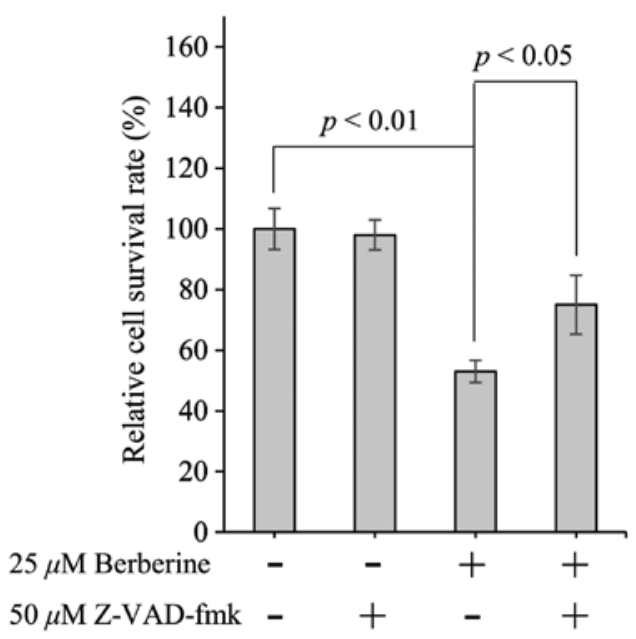

B

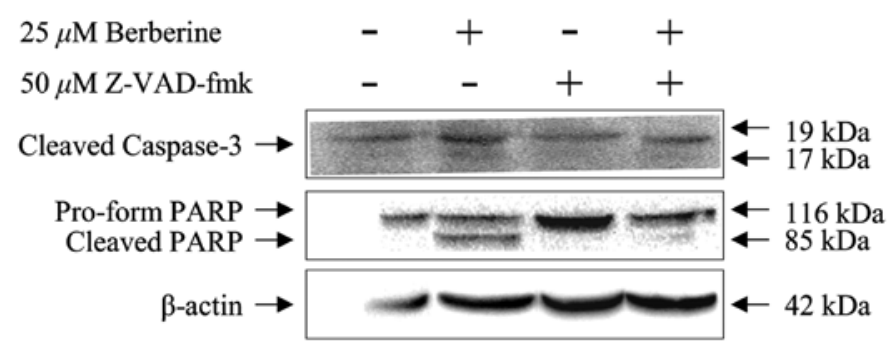

Figure 3. Berberine-induced apoptosis was dependent on the activation of caspases in FaDu cells. (A) Z-VAD-fmk, a pan-caspase inhibitor, reversed berberineinduced cell death in FaDu cells. (B) Activation of caspase-3 and PARP in FaDu cells treated with berberine was inhibited in the presence of Z-VAD-fmk. 
$\mathbf{A}$

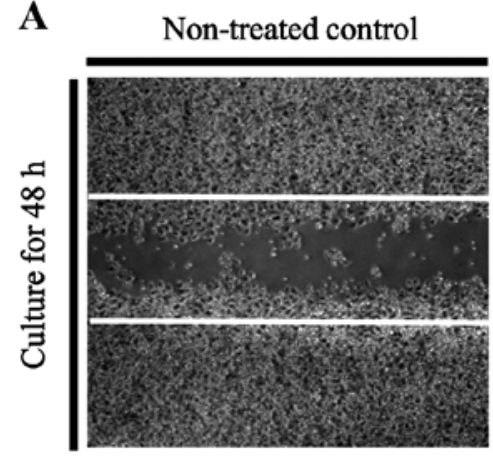

$12.5 \mu \mathrm{M}$ berberine

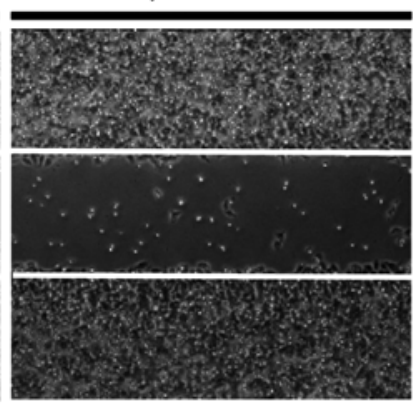

B

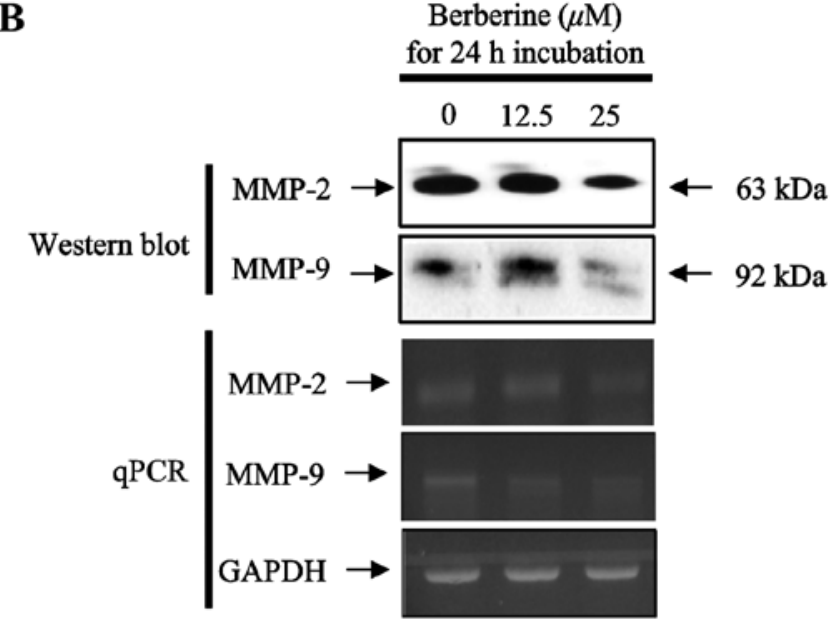

C

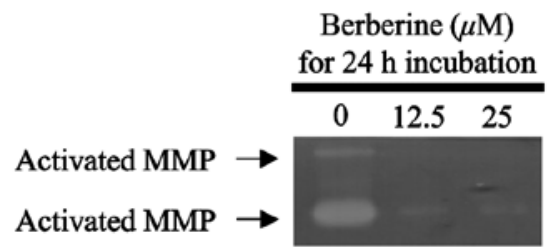

D

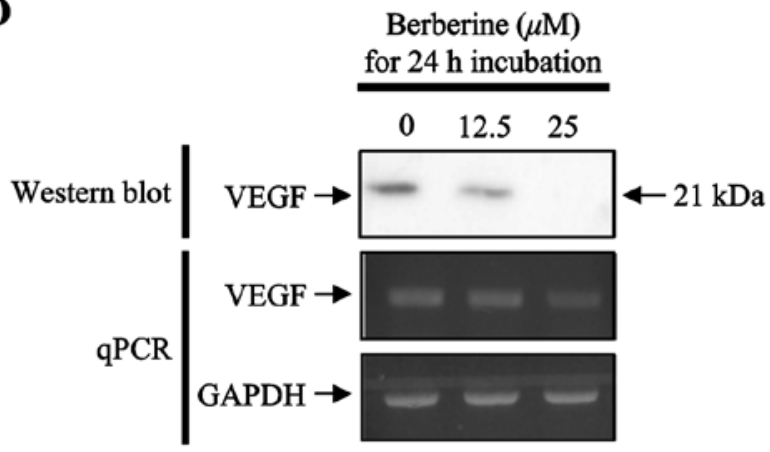

$\mathbf{E}$

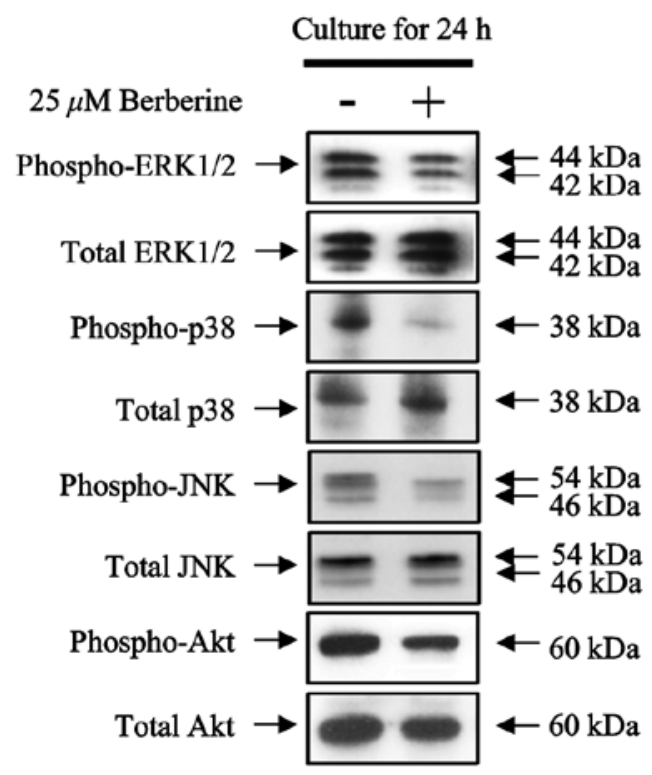

Figure 4. Berberine inhibits migration through the downregulation of MMP-2, MMP-9, and VEGF and through suppression of the phosphorylation of MAPK and PI3K/Akt signaling pathways in FaDu cells. (A) Berberine inhibited the migration of FaDu cells. (B and C) Berberine suppressed the expression and activation of MMPs associated with migration in FaDu cells. (D) The expression of VEGF was suppressed in FaDu cells treated with berberine. (E) The phosphorylation of MAPK and PI3K/Akt was suppressed in FaDu cells treated with berberine for $24 \mathrm{~h}$.

in the expression of caspase-3 and PARP. As shown in Fig. 3A, $25 \mu \mathrm{M}$ berberine decreased the viability of FaDu cells by $\sim 45 \%$ compared with that in untreated control cells. However, the viability of $\mathrm{FaDu}$ cells was partially recovered (by $\sim 74 \%$ ) in the presence of Z-VAD-fmk and berberine. Furthermore, the berberine-induced upregulation of cleaved caspase-3 was decreased by Z-VAD-fmk in FaDu cells. Subsequently, Z-VAD-fmk significantly suppressed berberine-induced cleavage of the pro-form of PARP in FaDu cells (Fig. 3B). Therefore, these data indicated that berberine-induced apoptosis in FaDu cells was regulated by the activation of caspases.

Berberine suppressed the migration of FaDu cells through the expression and activation of MMP-2 and MMP-9. Suppression of metastasis is a critical anticancer effect that should be considered during the development of chemotherapeutic agents. Therefore, we measured the migration of FaDu cells following treatment with berberine for 24 and $48 \mathrm{~h}$. As shown in Fig. 4A, migration was significantly inhibited in $\mathrm{FaDu}$ cells treated with $12.5 \mu \mathrm{M}$ berberine compared with that in untreated control cells. Furthermore, the protein and mRNA expression levels of MMP-2 and MMP-9, which are known to be associated with cancer cell migration, were decreased in FaDu cells treated with berberine in a concentration-dependent manner (Fig. 4B). Therefore, to verify the activation of MMPs secreted from berberine-treated FaDu cells into the conditioned medium, we performed the gelatin zymography assays. As shown in Fig. 4C, the clear bands formed by the digestion of gelatin, which is a substrate of MMP-2 and MMP-9, were less visible in FaDu cells treated with berberine in a concentration-dependent manner. Interestingly, the mRNA and protein expression levels of VEGF were significantly downregulated by berberine treatment in $\mathrm{FaDu}$ cells (Fig. 4D).

Berberine suppressed the phosphorylation of MAPK in FaDu cells. The expression of MMPs is closely associated with the activation of the MAPK signaling pathway in various types of cancer cells. Therefore, we investigated whether the activation of the MAPK signaling pathway was altered in FaDu cells 
treated with berberine. As shown in Fig. 4E, the phosphorylation of ERK1/2, p38, and JNK was significantly reduced in FaDu cells treated with $25 \mu \mathrm{M}$ berberine for $24 \mathrm{~h}$.

\section{Discussion}

Recent studies have shown that cancers of the oral cavity and pharynx have collectively become the six most common type of cancer worldwide, representing a global public health problem (27). Although clinical interventions for the management of oral cancer have improved, the 5-year survival rate for patients with oral cancer is only $\sim 50 \%$ (28).

Surgery is used to treat oral cancer and usually involves the removal of malignant and adjacent normal tissue in the oral cavity. However, such surgery may have various clinical side effects, including changes in the appearance and function of the oral cavity; this may in turn cause more severe emotional disturbance than that observed with other types of cancer (29). To reduce the clinical side effects caused by surgical approaches, chemotherapeutic approaches have become increasingly important in oral cancer. However, such chemotherapeutic approaches may have low efficacy and may cause additional adverse side effects, thereby restricting the use of many potential chemotherapeutic agents. Recent studies have focused on biologically active materials purified from medicinal herbs that are traditionally used in folk medicine and are considered biologically safe in order to develop chemotherapeutic agents with high efficacy and fewer side effects. According to the previously described prerequisites for chemotherapeutic agents, we reported that berberine did not affect the viability of normal human primary oral keratinocytes (23). Furthermore, berberine exhibited cytotoxic effects in KB oral cancer cells derived from an epidermal carcinoma of the oral cavity and caused significant cytotoxicity in $\mathrm{FaDu}$ cells, as shown in Fig. 1A and B. These data suggested that berberine may have fewer side effects by causing cell death specifically in cancer cells without affecting normal cells. However, the $\mathrm{IC}_{50}$ value of berberine in $\mathrm{FaDu}$ cells was $\sim 25 \mu \mathrm{M}$, which was 8-fold higher than that in KB cells, U937 human leukemic monocyte lymphoma cells, and B16 mouse melanoma cells $(23,30)$. Moreover, the $\mathrm{IC}_{50}$ value of berberine in SNU-5 human gastric carcinoma cells is $48 \mu \mathrm{M}$, which is $\sim 2$-fold higher than that in $\mathrm{FaDu}$ cells. Therefore, the $\mathrm{IC}_{50}$ value of berberine may exhibit tissue-type specificity.

Chromatin condensation caused by DNA fragmentation is a typical feature of apoptotic cells (31). In the present study, although we did not observe DNA fragmentation in $\mathrm{FaDu}$ cells treated with berberine, chromatin condensation was observed, as shown in Fig. 1C. Furthermore, FACS analysis using Annexin V and PI showed that the population of Annexin V-positive FaDu cells was significantly upregulated following treatment with berberine. Annexin V, a $\mathrm{Ca}^{2+}$-dependent phospholipid-binding protein, has high affinity for membrane phosphatidylserine translocated to the cell surface from the inner plasma membrane at early stage of apoptosis. However, apoptosis or necrosis occurring at the late stage of cell death is caused by the translocation of membrane phosphatidylserine, which precedes the loss of membrane integrity. Therefore, PI staining was performed to stain the membranes of dead and damaged cells to identify apoptotic cells in early and late stages. As shown in Fig. 1D, the apoptotic population was significantly increased by $55.21 \%$ in FaDu cells treated with $25 \mu \mathrm{M}$ berberine compared with that in untreated control cells. Interestingly, in FaDu cells treated with berberine for $24 \mathrm{~h}$, early-stage apoptotic cells accounted for $~ 54.49 \%$ of the population, while the percentage of late-stage apoptotic cells was much lower. Taken together, these data showed that apoptotic cell death was involved in berberine-induced $\mathrm{FaDu}$ cell death.

However, apoptosis (programmed cell death), is tightly regulated by the death receptor-mediated extrinsic pathway and the mitochondria-dependent intrinsic pathway (32). Furthermore, the selective modulation of both apoptotic signaling pathways could provide important insights into the targeting of apoptosis during the development of novel chemotherapeutic agents (11). Therefore, to examine the involvement of berberine-induced apoptotic signaling pathways in $\mathrm{FaDu}$ cells, we observed changes in the expression and activation of pro- and anti-apoptotic factors associated with death receptor-dependent extrinsic and mitochondria-dependent intrinsic apoptotic signaling pathways. In the death receptormediated extrinsic pathway, death receptor-specific ligands, such as FasL and TRAIL, trigger the sequential activation of pro-apoptotic factors, such as caspase-8, caspase-3, and PARP, thereby inducing cell death (33). Furthermore, activated caspase- 8 induces cleavage of cytosolic BH3 interacting-domain death agonist (BID) to truncated BID (tBID), resulting in loss of mitochondrial transmembrane potential through the insertion of Bax into the outer mitochondrial membrane; this triggers the mitochondria-dependent intrinsic apoptotic signaling pathway (34). Sequentially, the upregulation of Bad expression and the activation of caspase- 9 activate downstream pro-apoptotic factors, such as caspase-3 and PARP, thereby inducing cell death. Moreover, the downregulation of anti-apoptotic factors, such as Bcl-2 and Bcl-xL, accelerate mitochondria-dependent intrinsic apoptotic cell death $(35,36)$.

Therefore, we measured changes in the expression levels of death receptor ligands, such as FasL and TRAIL, which initiate not only the death receptor-dependent extrinsic apoptotic signaling pathway, but also the mitochondria-dependent intrinsic apoptotic signaling pathway through the activation of its downstream target caspase-8. As shown in Fig. 2A, the expression of death receptor ligands FasL and TRAIL was significantly upregulated in $\mathrm{FaDu}$ cells treated with berberine in a concentration-dependent manner. Subsequently, the upregulated death receptor ligands (i.e., FasL and TRAIL) initiated activation of the caspase cascade through the caspase-8/PARP axis, defined as the death receptor-dependent extrinsic apoptotic signaling pathway. Moreover, the activated caspase- 8 triggered the mitochondria-dependent intrinsic apoptotic signaling pathway, which involves upregulation or activation of pro-apoptotic factors, such as Bax, Bad, Apaf-1, and caspase-9, and downregulation of anti-apoptotic factors, such as Bcl-2 and Bcl-xL, as shown in Fig. 2B. Taken together, these data clearly demonstrated that berberine-induced apoptosis in $\mathrm{FaDu}$ cells was mediated by both the death receptor-dependent extrinsic and mitochondria-dependent intrinsic apoptotic signaling pathways. Similar to the our results in present study, Kim et al (24) reported that the berberine-induced apoptosis in KB oral 
cancer cells was mediated by both death receptor-dependent extrinsic and mitochondria-dependent intrinsic apoptotic signaling pathways triggered by the upregulation of the death ligand FasL.

However, the end stage of both apoptotic signaling pathways is mediated by activation of caspase-3 and PARP (Fig. 2C). Consistent with this, we observed activation of caspase- 3 using the cell-permeable fluorogenic caspase substrate PhiPhiLux caspase-3/-7 in FaDu cells treated with berberine, as shown in Fig. 2D. These data suggested that berberine-induced apoptosis in $\mathrm{FaDu}$ cells may depend on activation of the caspase cascade, a critical early step in apoptosis. As shown in Fig. 3A, the cytotoxic effects of berberine in FaDu cells were partially decreased in the presence of Z-VAD-fmk, a pan-caspase inhibitor. Furthermore, the activation of caspase-9 and its downstream target PARP was significantly decreased in FaDu cells co-treated with berberine and Z-VAD-fmk (Fig. 3B). These data further supported that berberine-induced apoptosis in $\mathrm{FaDu}$ cells was dependent on activation of the caspase cascade.

Interestingly, the expression of the tumor suppressor p53 was increased in berberine-treated FaDu cells in a concentration-dependent manner, as shown in Fig. 2B. Upregulation and activation of p53 lead to G1 phase cell cycle arrest (37) and cause initiation of apoptosis through induction of Bax (38) and p53 upregulated modulator of apoptosis (PUMA) expression in the context of DNA damage (39). Recent studies have reported that berberine-induced upregulation of the tumor suppressor p53 promotes cell cycle arrest in hepatocellular carcinoma cells (40) and prostate cancer cells (41). Therefore, although we did not observe berberine-induced cell cycle arrest in $\mathrm{FaDu}$ cells in the present study, cell cycle arrest may be induced in FaDu cells treated with berberine. Further studies are required to examine these additional potential anticancer effects of berberine.

In addition, recent studies have suggested that p53 may contribute to the regulation of cell invasion and migration (42); indeed, p53 mutations cause loss of cell growth suppression and increase cell migration and invasion (43-45). Therefore, we measured the migration of FaDu cells in the presence of berberine. As shown in Fig. 4A, berberine significantly suppressed the migration of FaDu cells compared with that in untreated control. Furthermore, the expression and activation of MMPs, such as MMP-2 and MMP-9, which are associated with cancer cell migration, were significantly suppressed in FaDu cells treated with berberine in a concentration-dependent manner, as shown in Fig. 4B and C. Signals from tumor cells can promote the expression and activation of MMPs, which degrade the extracellular membrane, thereby initiating cancer cell migration. In particular, the expression levels of MMP-2 and MMP-9 are closely associated with the development and progression of cancer (46). Therefore, MMP expression has interesting implications in the potential use of MMP inhibitors as chemotherapeutic agents. In the present study, our results clearly suggested that berberine inhibited the migration of FaDu cells through suppression of MMP expression and activation.

In the present study, we showed the berberine reduced the mRNA and protein levels of VEGF, a representative growth factor associated with angiogenesis, as shown in Fig. 4D.
Angiogenesis plays a crucial role in the development of many types of cancer (47). In particular, the upregulation of VEGF not only promotes endothelial cell proliferation and migration, but also increases tumor size by promoting the formation of new blood vessels and increasing the permeability of existing blood vessels (47). Therefore, anti-angiogenesis therapy may be a promising strategy in the development of chemotherapeutic agents. Moreover, although we did not observe the biological relationship between p53 and the expression of VEGF in the present study, others have reported that p53 indirectly downregulates VEGF expression $(48,49)$. Previously, we showed that berberine induced upregulation of p53 in FaDu cells, as shown in Fig. 2B. Therefore, berberine-induced p53 expression may be associated with changes in the expression of VEGF in $\mathrm{FaDu}$ cells, representing one of the anticancer effects of berberine.

Next, to verify the cellular signaling pathways associated with the anticancer activities of berberine, we measured changes in the expression and activation of MAPK and PI3K/Akt signaling pathway components in FaDu cells treated with berberine. Phorbol 12-myristate (PMA) has been shown to induce MMP expression (50) through activation of the PI3K/Akt and MAPK signaling pathways (51). Recently, Im et al reported that inhibition of MAPK activation suppresses PMA-induced MMP-9 expression in MCF-7 human breast cancer cells (53). Furthermore, Lin et al showed that resveratrol suppresses 12-O-tetradecanoylphorbol13-acetate (TPA)-induced MMP-9 expression through inhibition of the MAPK pathway in oral cancer cells (54). Although the signaling pathways that lead to upregulation of MMP expression are still incompletely understood, recent studies have suggested that the MAPK signaling pathway may regulate the expression of MMPs associated with cancer cell migration. Thus, the regulation of MMP expression using specific chemical inhibitors or natural compounds targeting cellular signaling pathways, such as the PI3K/Akt and MAPK pathways, could be used as potential chemotherapeutic agents. In the present study, we showed that the phosphorylation of MAPKs, such as ERK1/2 and p38, as well as that of PI3K/Akt was significantly suppressed in FaDu cells treated with berberine for $24 \mathrm{~h}$; interestingly, the phosphorylation of JNK was not inhibited, as shown in Fig. 4E. Therefore, these data suggested that berberine suppressed the expression of MMPs through inhibition of MAPK and PI3K/Akt signaling pathways in FaDu cells.

In addition, recent studies have reported the suppression of angiogenesis by targeting VEGF through the PI3K/Akt and MAPK signaling pathways in various types of cancer $(54,55)$. Therefore, the PI3K/Akt and MAPK signaling pathways are closely associated with the regulation of VEGF expression in cancer. Furthermore, the inhibition of PI3K/Akt and MAPK signaling pathways may suppress tumor growth by inhibition of angiogenesis through the downregulation of VEGF expression (56).

In conclusion, we demonstrated the anticancer effects of berberine in FaDu cells. In particular, berberine induced apoptosis, suppressed migration through the downregulation of MMP expression, and exerted anti-angiogenic effects through the downregulation of VEGF expression. Taken together, these findings suggested that berberine may be a promising candidate chemotherapeutic agent for the treatment of HNSCC. 


\section{Acknowledgements}

This study was supported by a research fund from the Chosun University Dental Hospital, 2015.

\section{References}

1. Sturgis EM and Miller RH: Second primary malignancies in the head and neck cancer patient. Ann Otol Rhinol Laryngol 104: 946-954, 1995.

2. Rothman KJ: The effect of alcohol consumption on risk of cancer of the head and neck. Laryngoscope 88 (Suppl 8): 51-55, 1978.

3. Maier H, Dietz A, Gewelke U, Heller WD and Weidauer H: Tobacco and alcohol and the risk of head and neck cancer. Clin Investig 70: 320-327, 1992.

4. Rothman KJ: Epidemiology of head and neck cancer. Laryngoscope 88: 435-438, 1978.

5. Lin YS, Jen YM, Wang BB, Lee JC and Kang BH: Epidemiology of oral cavity cancer in Taiwan with emphasis on the role of betel nut chewing. ORL J Otorhinolaryngol Relat Spec 67: 230-236, 2005.

6. Mannarini L, Kratochvil V, Calabrese L, Gomes Silva L, Morbini P, Betka J and Benazzo M: Human papilloma virus (HPV) in head and neck region: Review of literature. Acta Otorhinolaryngol Ital 29: 119-126, 2009.

7. Jemal A, Bray F, Center MM, Ferlay J, Ward E and Forman D: Global cancer statistics. CA Cancer J Clin 61: 69-90, 2011.

8. Lacko M, Braakhuis BJ, Sturgis EM, Boedeker CC, Suárez C, Rinaldo A, Ferlito A and Takes RP: Genetic susceptibility to head and neck squamous cell carcinoma. Int J Radiat Oncol Biol Phys 89: 38-48, 2014.

9. Park MR, Kim SG, Cho IA, Oh D, Kang KR, Lee SY, Moon SM, Cho SS, Yoon G, Kim CS, et al: Licochalcone-A induces intrinsic and extrinsic apoptosis via ERK1/2 and p38 phosphorylation-mediated TRAIL expression in head and neck squamous carcinoma FaDu cells. Food Chem Toxicol 77: 34-43, 2015.

10. Fesik SW: Promoting apoptosis as a strategy for cancer drug discovery. Nat Rev Cancer 5: 876-885, 2005.

11. Renehan AG, Booth C and Potten CS: What is apoptosis, and why is it important? BMJ 322: 1536-1538, 2001.

12. Khan KH, Blanco-Codesido M and Molife LR: Cancer therapeutics: Targeting the apoptotic pathway. Crit Rev Oncol Hematol 90: 200-219, 2014

13. Luqmani YA: Mechanisms of drug resistance in cancer chemotherapy. Med Princ Pract 14 (Suppl 1): 35-48, 2005.

14. Ni WJ, Ding HH and Tang LQ: Berberine as a promising anti-diabetic nephropathy drug: An analysis of its effects and mechanisms. Eur J Pharmacol 760: 103-112, 2015.

15. Hu Z, Jiao Q, Ding J, Liu F, Liu R, Shan L, Zeng H, Zhang J and Zhang W: Berberine induces dendritic cell apoptosis and has therapeutic potential for rheumatoid arthritis. Arthritis Rheum 63: 949-959, 2011.

16. Xiao HB, Sun ZL, Zhang HB and Zhang DS: Berberine inhibits dyslipidemia in C57BL/6 mice with lipopolysaccharide induced inflammation. Pharmacol Rep 64: 889-895, 2012.

17. Li Z, Geng YN, Jiang JD and Kong WJ: Antioxidant and anti-inflammatory activities of berberine in the treatment of diabetes mellitus. Evid Based Complement Alternat Med 2014: 289264, 2014.

18. Tan Y, Tang Q, Hu BR and Xiang JZ: Antioxidant properties of berberine on cultured rabbit corpus cavernosum smooth muscle cells injured by hydrogen peroxide. Acta Pharmacol Sin 28: 1914-1918, 2007.

19. Zhang X, Zhao Y, Zhang M, Pang X, Xu J, Kang C, Li M, Zhang C, Zhang Z, Zhang Y, et al: Structural changes of gut microbiota during berberine-mediated prevention of obesity and insulin resistance in high-fat diet-fed rats. PLoS One 7: e42529, 2012.

20. Kim S, Choi JH, Kim JB, Nam SJ, Yang JH, Kim JH and Lee JE: Berberine suppresses TNF-alpha-induced MMP-9 and cell invasion through inhibition of AP-1 activity in MDA-MB-231 human breast cancer cells. Molecules 13: 2975-2985, 2008.

21. Li J, Cao B, Liu X, Fu X, Xiong Z, Chen L, Sartor O, Dong Y and Zhang H: Berberine suppresses androgen receptor signaling in prostate cancer. Mol Cancer Ther 10: 1346-1356, 2011.
22. Lin JP, Yang JS, Chang NW, Chiu TH, Su CC, Lu KW, Ho YT, Yeh CC, Mei-Dueyang, Lin HJ, et al: GADD153 mediates berberine-induced apoptosis in human cervical cancer Ca ski cells. Anticancer Res 27: 3379-3386, 2007.

23. Lin JP, Yang JS, Wu CC, Lin SS, Hsieh WT, Lin ML, Yu FS, Yu CS, Chen GW, Chang YH, et al: Berberine induced down-regulation of matrix metalloproteinase-1, -2 and -9 in human gastric cancer cells (SNU-5) in vitro. In Vivo 22: 223-230, 2008.

24. Kim JS, Oh D, Yim MJ, Park JJ, Kang KR, Cho IA, Moon SM, Oh JS, You JS, Kim CS, et al: Berberine induces FasL-related apoptosis through p38 activation in KB human oral cancer cells. Oncol Rep 33: 1775-1782, 2015.

25. Kuo CL, Chi CW and Liu TY: Modulation of apoptosis by berberine through inhibition of cyclooxygenase-2 and Mcl-1 expression in oral cancer cells. In Vivo 19: 247-252, 2005.

26. Jin P, Zhang $\mathrm{C}$ and $\mathrm{Li} \mathrm{N}$ : Berberine exhibits antitumor effects in human ovarian cancer cells. Anticancer Agents Med Chem 15: 511-516, 2015.

27. Kim JS, Ellman MB, An HS, Yan D, van Wijnen AJ, Murphy G, Hoskin DW and Im HJ: Lactoferricin mediates anabolic and anti-catabolic effects in the intervertebral disc. J Cell Physiol 227: 1512-1520, 2012

28. Warnakulasuriya S: Global epidemiology of oral and oropharyngeal cancer. Oral Oncol 45: 309-316, 2009.

29. Parkin DM, Bray F, Ferlay J and Pisani P: Global cancer statistics, 2002. CA Cancer J Clin 55: 74-108, 2005.

30. Silverman S Jr: Oral cancer: Complications of therapy. Oral Surg Oral Med Oral Pathol Oral Radiol Endod 88: 122-126, 1999.

31. Letasiová S, Jantová S, Cipák L and Múcková M: Berberine-antiproliferative activity in vitro and induction of apoptosis/necrosis of the U937 and B16 cells. Cancer Lett 239: 254-262, 2006.

32. Oberhammer FA, Hochegger K, Fröschl G, Tiefenbacher R and Pavelka M: Chromatin condensation during apoptosis is accompanied by degradation of lamin $\mathrm{A}+\mathrm{B}$, without enhanced activation of cdc2 kinase. J Cell Biol 126: 827-837, 1994.

33. Hensley P, Mishra M and Kyprianou N: Targeting caspases in cancer therapeutics. Biol Chem 394: 831-843, 2013.

34. Ikner A and Ashkenazi A: TWEAK induces apoptosis through a death-signaling complex comprising receptor-interacting protein 1 (RIP1), Fas-associated death domain (FADD), and caspase-8. J Biol Chem 286: 21546-21554, 2011.

35. Li H, Zhu H, Xu CJ and Yuan J: Cleavage of BID by caspase 8 mediates the mitochondrial damage in the Fas pathway of apoptosis. Cell 94: 491-501, 1998.

36. Fischer B, Coelho D, Dufour P, Bergerat JP, Denis JM, Gueulette J and Bischoff P: Caspase 8-mediated cleavage of the pro-apoptotic BCL-2 family member BID in p53-dependent apoptosis. Biochem Biophys Res Commun 306: 516-522, 2003.

37. Zhu J, Xiong L, Yu B and Wu J: Apoptosis induced by a new member of saponin family is mediated through caspase-8-dependent cleavage of Bcl-2. Mol Pharmacol 68: 1831-1838, 2005.

38. Harper JW, Adami GR, Wei N, Keyomarsi K and Elledge SJ: The p21 Cdk-interacting protein Cip1 is a potent inhibitor of G1 cyclin-dependent kinases. Cell 75: 805-816, 1993.

39. Chipuk JE, Kuwana T, Bouchier-Hayes L, Droin NM, Newmeyer DD, Schuler M and Green DR: Direct activation of Bax by 53 mediates mitochondrial membrane permeabilization and apoptosis. Science 303: 1010-1014, 2004.

40. Wang P, Yu J and Zhang L: The nuclear function of $\mathrm{p} 53$ is required for PUMA-mediated apoptosis induced by DNA damage. Proc Natl Acad Sci USA 104: 4054-4059, 2007.

41. Wang N, Zhu M, Wang X, Tan HY, Tsao SW and Feng Y: Berberine-induced tumor suppressor p53 up-regulation gets involved in the regulatory network of miR-23a in hepatocellular carcinoma. Biochim Biophys Acta 1839: 849-857, 2014.

42. Wang Y, Liu Q, Liu Z, Li B, Sun Z, Zhou H, Zhang X, Gong Y and Shao C: Berberine, a genotoxic alkaloid, induces ATM-Chk1 mediated G2 arrest in prostate cancer cells. Mutat Res 734: 20-29, 2012.

43. Wu JX, Zhang DG, Zheng JN and Pei DS: Rap2a is a novel target gene of p53 and regulates cancer cell migration and invasion. Cell Signal 27: 1198-1207, 2015.

44. Boudreau HE, Casterline BW, Burke DJ and Leto TL: Wild-type and mutant p53 differentially regulate NADPH oxidase 4 in TGF- $\beta$-mediated migration of human lung and breast epithelial cells. Br J Cancer 110: 2569-2582, 2014.

45. Momota H, Narita Y, Matsushita Y, Miyakita Y and Shibui S: p53 abnormality and tumor invasion in patients with malignant astrocytoma. Brain Tumor Pathol 27: 95-101, 2010. 
46. Muller PA, Caswell PT, Doyle B, Iwanicki MP, Tan EH, Karim S, Lukashchuk N, Gillespie DA, Ludwig RL, Gosselin P, et al: Mutant p53 drives invasion by promoting integrin recycling. Cell 139: 1327-1341, 2009.

47. Chambers AF and Matrisian LM: Changing views of the role of matrix metalloproteinases in metastasis. J Natl Cancer Inst 89: 1260-1270, 1997.

48. Tomao F, Papa A, Rossi L, Zaccarelli E, Caruso D, Zoratto F, Benedetti Panici P and Tomao S: Angiogenesis and antiangiogenic agents in cervical cancer. Onco Targets Ther 7: 2237-2248, 2014.

49. Mukhopadhyay D, Tsiokas L and Sukhatme VP: Wild-type p53 and $\mathrm{v}$-Src exert opposing influences on human vascular endothelial growth factor gene expression. Cancer Res 55: 6161-6165, 1995.

50. Qin G, Kishore R, Dolan CM, Silver M, Wecker A Luedemann CN, Thorne T, Hanley A, Curry C, Heyd L, et al: Cell cycle regulator E2F1 modulates angiogenesis via p53-dependent transcriptional control of VEGF. Proc Natl Acad Sci USA 103: 11015-11020, 2006.

51. Ehrenfeld P, Conejeros I, Pavicic MF, Matus CE, Gonzalez CB, Quest AF, Bhoola KD, Poblete MT, Burgos RA and Figueroa CD: Activation of kinin $\mathrm{B} 1$ receptor increases the release of metalloproteases-2 and -9 from both estrogen-sensitive and -insensitive breast cancer cells. Cancer Lett 301: 106-118, 2011.
52. Eberhardt W, Huwiler A, Beck KF, Walpen S and Pfeilschifter J: Amplification of IL-1 beta-induced matrix metalloproteinase-9 expression by superoxide in rat glomerular mesangial cells is mediated by increased activities of NF-kappa B and activating protein-1 and involves activation of the mitogen-activated protein kinase pathways. J Immunol 165: 5788-5797, 2000.

53. Im NK, Jang WJ, Jeong CH and Jeong GS: Delphinidin suppresses PMA-induced MMP-9 expression by blocking the NF- $\kappa \mathrm{B}$ activation through MAPK signaling pathways in MCF-7 human breast carcinoma cells. J Med Food 17: 855-861, 2014.

54. Lin FY, Hsieh YH, Yang SF, Chen CT, Tang CH, Chou MY, Chuang YT, Lin CW and Chen MK: Resveratrol suppresses TPA-induced matrix metalloproteinase- 9 expression through the inhibition of MAPK pathways in oral cancer cells. J Oral Pathol Med: Nov 17, 2014 (Epub ahead of print). doi: 10.1111/jop.12288.

55. Tong Q, Qing Y, Wu Y, Hu X, Jiang L and Wu X: Dioscin inhibits colon tumor growth and tumor angiogenesis through regulating VEGFR 2 and AKT/MAPK signaling pathways. Toxicol Appl Pharmacol 281: 166-173, 2014

56. Wang W, Ren F, Wu Q, Jiang D, Li H and Shi H: MicroRNA-497 suppresses angiogenesis by targeting vascular endothelial growth factor A through the PI3K/AKT and MAPK/ERK pathways in ovarian cancer. Oncol Rep 32: 2127-2133, 2014. 